\title{
Estudo de Caso Sobre a Inclusão de Alunos com Deficiência no ENSINO SUPERIOR
}

\author{
Case Study of InClusion of Students With Disabilities in Higher \\ EDUCATION
}

\author{
Emerson Rodrigues DUARTE ${ }^{1}$ \\ Carla Beatriz da Silva RAFAEL ${ }^{2}$ \\ Juliana Fernandes FILGUEIRAS ${ }^{3}$ \\ Clara Mockdece NEVES ${ }^{4}$ \\ Maria Elisa Caputo FERREIRA 5
}

\begin{abstract}
RESUMO: o objetivo do presente estudo foi levantar o número de alunos auto-declarados com deficiência em processo de inclusão no ensino superior nas instituiçóes públicas e privadas de Juiz de Fora. Tal temática merece destaque vista as políticas implementadas pelo Governo Federal de acesso ao Ensino Superior, como o ProUni, o REUNI e o FIES a fim de ampliar o nível de escolarização da população. Assim, foram pesquisadas 11 Instituiçóes de Ensino Superior de Juiz de Fora, sendo dez privadas e uma pública. Para tanto, foram entrevistados os coordenadores de cursos que contavam, no período da coleta de dados, com alunos auto-declarados com deficiência, regularmente matriculados e frequentando o curso. As entrevistas foram submetidas à análise de conteúdo. Foi possível perceber que existem, atualmente, 45 alunos com deficiência matriculados e frequentes em cursos de ensino superior da cidade. A rede privada representa $82,2 \%$ desse total e a rede pública federal, 17,8\%. Portanto, é de grande relevância a concretização de estudos que visem ampliar as informaçóes sobre a inclusão de pessoas com deficiência na rede de ensino brasileira. Isso possibilitará o entendimento do processo de dificuldades, luta e superação desses estudantes até a chegada ao Ensino Superior. Esta questão tem sido importante para dar assistência aos profissionais envolvidos na ação pelo direito das pessoas com deficiência e o acesso dos mesmos, em especial, no meio educacional.
\end{abstract}

PALAVRAS-CHAVE: Educação Especial. Pessoa com Deficiência. Ensino Superior.

ABSTRACT: The aim of this study was to research the number of self-declared students with disabilities in the process of inclusion in public and private higher education institutions of Juiz de Fora. This theme merits investigation because of the policies implemented by the Federal Government Access to Higher Education that aim to increase the level of schooling for the population, i.e. ProUni, REUNI and FIES. Eleven higher education institutions of Juiz de Fora were surveyed, of which ten were private and one public. The interviewees were the supervisors of courses in which self-declared students with disabilities were enrolled, and attended regularly, at the time when data was collected. Subsequently, the interviews were analyzed by content analysis. The data showed that there are currently 45 students with disabilities enrolled who regularly attend classes in higher education courses in the city. The private institutions represent $82.2 \%$ of this total and the federal public university includes $17.8 \%$. It is deemed greatly relevant to implement further studies that aim to increase information about the inclusion of people with disabilities in the Brazilian school system. This will enable better understanding of these students' process of facing difficulties, advocacy struggles and overcoming barriers until they achieve entrance into the university. This issue has been important in assisting professionals involved in advocacy for the rights of people with disabilities, especially regarding access to educational systems.

KEYWORD: Special Education. People with Disabilities. Higher Education.

\footnotetext{
${ }^{1}$ Doutorando em Psicologia da Universidade Federal de Juiz de Fora. Juiz de Fora - MG. duarte.emerson@terra.com.br

${ }^{2}$ Mestranda em Educação Física da Universidade Federal de Juiz de Fora. Juiz de Fora - MG. carla2003rafael@yahoo.com.br

${ }^{3}$ Mestranda em Psicologia da Universidade Federal de Juiz de Fora. Juiz de Fora - MG. eujuly90@hotmail.com

${ }^{4}$ Mestranda em Psicologia da Universidade Federal de Juiz de Fora. Juiz de Fora - MG. clarinhamockdece@hotmail.com

${ }^{5}$ Pós-Doutora em Educação. Professora da Faculdade de Educação Física da Universidade Federal de Juiz de Fora. Juiz de Fora -

MG. caputoferreira@terra.com.br
} 


\section{INTRODUÇÁo}

Esse artigo tem como tema principal a inclusão de pessoas com deficiência no ensino superior. Tal temática merece destaque vista as políticas implementadas pelo Governo Federal de acesso ao Ensino Superior, como o Pro-Uni, o REUNI e o FIES, com o objetivo de ampliar o nível de escolarizaçáo da população.

No mundo contemporâneo, a inclusão do aluno com deficiência representa desafio, desde a modalidade de Educação Infantil até o Ensino Superior, em instituiçôes públicas e privadas. No Brasil, as estatísticas oficiais, estudos e pesquisas, elucidam principalmente a condição desse alunado em processo de inclusão na educação básica subsidiados pelas Declaraçóes de Educação para Todos (UNESCO, 1990) e de Salamanca (ONU, 1994).

Todavia, pouco se tem documentado sobre a inclusão de pessoas com deficiência no ensino superior, indicando uma carência de reflexóes, estudos e estatísticas dificultando, assim, a formulação de políticas públicas que contemplem açóes que avancem para uma educaçáo inclusiva também no ensino superior. Entre estes poucos, podemos citar, atualmente, estudiosos como: Silva et al. (2012); Vianna, Tardelli e Almeida (2012); Inajara, Santana e Silva (2010); Ferreira (2007); Pereira (2007); Chahini (2006); Mansini e Bazon (2006); Pellegrini (2006); Perini (2006) e Rodrigues (2004). Estes autores se dedicaram a estudar este paradigma educacional da sociedade pós-moderna apontado valores, processos, caminhos e dificuldades a serem considerados a respeito das possibilidades de acesso e permanência de pessoas com deficiência no ensino superior.

Especificamente em Juiz de Fora/MG, náo foi possível encontrar dados referentes ao número de pessoas com deficiência em seus diversos cursos de graduação sequer na única universidade pública da cidade tampouco nas faculdades privadas.

Por esta lacuna de dados locais, contou-se apenas com valores nacionais que apontam para o crescimento do número de matrícula de alunos com deficiência no ensino superior de uma forma geral. Segundo o Censo da Educação Superior MEC/INEP (BRASIL, 2012), havia, neste ano, 22.455 matrículas de alunos com deficiência no ensino superior, sendo deste total, 16.790 nas Instituiçóes Privadas de Ensino Superior e 4.437 nas Instituições Federais de Ensino Superior.

É neste sentido que este estudo se justifica, visto que há uma necessidade contemporânea de estabelecer processos e metodologias em políticas educacionais, que considerem sobremaneira a diversidade humana e contemplem o Ensino Superior. Assim, na cidade de Juiz de Fora, há uma barreira para o estabelecimento de tais políticas educacionais, visto que náo foram encontrados dados epidemiológicos que abranjam a inclusão de pessoas com deficiência no Ensino Superior. Estes dados tornam-se, portanto, importantes e necessários para o desenvolvimento de estratégias e açóes em políticas públicas educacionais. Neste sentido, apresenta-se essa proposta de estudo no intuito de levantar informaçóes relevantes sobre a inclusão de alunos com deficiência nas Instituições de Ensino Superior (IES) de Juiz de Fora.

Portanto, este estudo teve como objetivo relatar a inclusão de pessoas com deficiência nas IES pública e privadas de Juiz de Fora/MG. 


\subsection{A InClusão/EXCluSÃo EdUCACIONAL dAS PESSOAS COM DEFICIÊNCIA}

Podemos considerar o conceito de inclusão muito recente se comparado a trajetória secular de exclusão. Referindo-se aos estudos de Machado, Tres e Oliveira (2011), Carmo (2008), Ferreira e Guimarães (2003) e Mazzota (1996), é possível perceber que ao longo dos séculos houve diversas mudanças conceituais sobre a deficiência e sobre o tratamento dispensado pela sociedade às pessoas que apresentavam deficiências físicas, intelectuais ou sensoriais. Para se ter uma ideia, na Antiguidade estas pessoas eram exterminadas, pois se acreditava que eram amaldiçoadas. Somente na Idade Média, este comportamento começou a mudar, devido à influência da Igreja Católica, que considerava todos como criaturas de Deus, independente de possuir ou não uma deficiência. No entanto, elas deixaram de ser mortas para serem segregadas e depender da caridade alheia para sua sobrevivência.

Com a intenção de romper este paradigma de segregação e adotar as ideias de normalização, foi criado o conceito de Integração, referente à necessidade de modificar a pessoa com deficiência - e não a sociedade - de forma que esta pudesse assemelhar-se, o máximo possível, aos demais, para ser inserida e integrada no convívio social.

Entretanto, como afirmam Ferreira e Guimarães (2003) e Mazzota (1996), normalizar o indivíduo com deficiência passou a não fazer sentido. $\mathrm{O}$ conceito de normalidade é muito relativo e subjetivo. Assim, esclarece Oliveira:

O surgimento de terminologias ligadas à Educação Especial entre elas a integração, a normalização, a inclusão, a diversidade, e outras tantas, refletem a sobrecarga que carrega todo aquele que é diferente, que não se encaixa a modelos pré-estabelecidos que o levem a fazer parte de grupos homogêneos, que se autodeterminam ser iguais perante outros considerados diferentes (OLIVEIRA, 2012, p. 2).

Diante disso, chegou-se à conclusão de que a sociedade também teria sua parcela de contribuiçãao ao processo de inserção das pessoas com deficiência. Os sistemas sociais, que durante séculos não contemplaram as necessidades específicas provenientes da diversidade humana, teriam que se transformar de modo a atender a todos. O processo deve ser bidirecional. Diante destas constatações e das inúmeras mudanças que vemos hoje eclodir na sociedade, surge o movimento da inclusão, que é consequência da visão social de um mundo democrático, onde se anseia respeitar direitos e deveres de todos, independente das diferenças de cada um. A limitação de uma pessoa não pode diminuir seus direitos. As pessoas com deficiência são cidadãos e fazem parte da sociedade e esta deve se preparar para lidar com a diversidade humana.

A “escolarização" é fundamental na constituição do indivíduo que vive em uma sociedade como a nossa, ainda marcada pela exclusão, fracasso e o abandono em todos os níveis de ensino. De fato, essa falha significa um grave impedimento da apropriação do saber sistematizado, da construção de funções psicológicas mais sofisticadas, de instrumentos de atuação no meio social e de transformação do sujeito e das condições para a construção de novos conhecimentos (REGO, 2003). 


\subsection{A inClusáo de pessoas com deficiênCIas no Ensino Superior}

Embora sejamos a favor da luta pela inclusão escolar das pessoas com deficiência, reconhecemos que os sujeitos envolvidos sofrem todos os tipos de descriminação e de imposição de uma sociedade que os elimina sendo falsa a concepção de que caminhamos rumo à igualdade de oportunidades (PERINI, 2006, p. 111).

Assevera-se que o pleno acesso e a permanência da pessoa com deficiência na escola não é ainda uma realidade. Diversos fatores culturais, políticos e sociais ainda contribuem para a manutençáo desse quadro de dificuldade, incluindo a negaçáo do direito de acesso à educação, podendo ser este um dos fatores que contribuem para o baixo índice de pessoas com deficiência inseridas no mercado de trabalho e/ou com rendimentos inferiores aos das pessoas sem deficiência (FERREIRA; DUARTE, 2010).

Assim, pode-se considerar que o fato de estar "dentro" da sala de aula não implica, necessariamente, que os alunos com deficiência estejam incluídos nos processos de ensino e de aprendizagem, pois, para isso acontecer, eles precisam se mobilizar e, de fato, absorverem os conteúdos escolares (GOMES; LIMA, 2006).

Não se pode esquecer ainda, que o desafio da inclusão no ensino superior passa por decisóes que extrapolam os muros das universidades públicas. É preciso considerar que a universidade pública brasileira não pode ser tomada como a única responsável por este processo, mas como parte integrante da implementaçáo de políticas públicas que garantam apoio financeiro às açôes e iniciativas neste contexto. Paralelamente a essa situação, as IES Federais precisam estar cientes da importância de expor às instâncias governamentais as limitaçóes que enfrentam e apontar encaminhamentos que devem ser tomados para que haja a garantia de acesso, ingresso e permanência desses estudantes, pois contam com profissionais das mais diversas áreas do conhecimento, que podem contribuir com ensino, pesquisa e extensão na área das necessidades educacionais especiais (MOREIRA, 2005). Também Mantoan e colaboradores expressam-se sobre esse tema afirmando que:

Resistimos à inclusão escolar porque ela nos faz lembrar que temos uma dívida a saldar em relação aos alunos que excluímos, por motivos muitas vezes banais e inconsistentes, apoiados por uma organizaçáo pedagógica escolar que se destina a alunos ideais, padronizados por uma concepçáo de normalidade e de deficiência arbitrariamente definida (MANTOAN et al., 2011, p. 78).

Acredita-se que com a inclusão, não só a pessoa com deficiência é beneficiada, mas todos aqueles envolvidos neste processo educacional. A convivência com a diversidade humana favorece a construção de novas relaçôes e experiências tấo indispensáveis e fundamentais na formação contemporânea humana e no desenvolvimento dos professores, profissionais e alunos, ampliando para eles a compreensão dos conceitos de justiça e direito (LIMA, 2007).

\section{Metodologia}

\subsection{Critérios éticos}

Esta pesquisa foi aprovada pelo Comitê de Ética em Pesquisa com Seres Humanos da Universidade Federal de Juiz de Fora. Foi solicitada autorização a todas as IES pesquisadas e o 
Termo de Consentimento Livre e Esclarecido (TCLE) foi entregue a todos participantes. Todas as entrevistas foram gravadas em mídia digital e posteriormente transcritas na sua íntegra. Foi mantido sigilo nominal de todas instituiçóes e entrevistados. Todo material se encontra arquivado, ficando disponível por cinco anos. Após este período, será destruído.

\subsection{Participantes}

A cidade de Juiz de Fora/MG contava, na fase de coleta dos dados, com 11 IES, sendo 10 privadas e uma pública. Ao todo, essas instituiçóes contavam com 116 coordenadores de cursos, dos quais 71 pertenciam à rede privada e 35, à rede pública.

Entretanto, para serem incluídas como participantes da presente pesquisa, foram consideradas apenas as IES que contavam com alunos com deficiência em processo de inclusão, regularmente matriculados e frequentes no ano de 2009. Dessa forma, foram entrevistados 21 coordenadores de oito IES particulares das 10 iniciais, e 35 coordenadores dos cursos oferecidos pela IES pública.

\subsection{INSTRUMENTOS}

Como principal instrumento de coleta de dados, foi elaborado um roteiro para as entrevistas o qual foi avaliado por uma banca de cinco doutores na área de inclusão. As sugestóes de alteraçóes foram acatadas para a construção da versão final desse instrumento.

Além disso, foi aplicado um questionário sociodemográfico, contendo informaçôes preliminares e importantes do encontro, tais como: data, local, instituição e curso.

\subsection{Procedimentos}

Inicialmente, com o objetivo de levantar a existência de alunos com deficiência regularmente matriculados e frequentes nas 10 IES particulares, contataram-se as diretorias, secretarias ou núcleos de apoio psicopedagógicos. Na IES pública pesquisada, optou-se por entrevistar os 35 coordenadores de todos os cursos de graduação, devido à inexistência de informações a respeito da inclusão de alunos com deficiência. Nenhum órgão oficial tinha qualquer tipo de levantamento para quantificar e caracterizar os alunos com deficiência em processo de inclusão. Assumiu-se a escolha em entrevistar os coordenadores de todos os cursos por acreditar que estes têm uma visão processual e longitudinal do processo de inclusão da pessoa com deficiência nos cursos sob sua responsabilidade.

Após o contato inicial, os objetivos e procedimentos da pesquisa foram esclarecidos e, com o consentimento para participação voluntária, foram agendadas entrevistas com os respectivos coordenadores de cursos. Foi solicitada formalmente a autorização para a gravação das entrevistas as quais foram realizadas em apenas uma sessão e em horários e locais variados. Procurou-se atender ao que era mais conveniente para o entrevistado, em geral, nas salas dos coordenadores.

As perguntas e os temas centrais serviram como diretrizes as quais possibilitaram que o sujeito desenvolvesse seu próprio discurso. Sendo assim, as questốes elaboradas não 
foram respondidas uma a uma, pois isso poderia limitar a narrativa do entrevistado, além de interromper o processo de pensamento. A coleta de dados foi realizada entre os meses de maio e junho de 2009.

\subsection{ANÁLISE DOS DADOS}

Os dados quantitativos percebidos a partir das transcriçóes das entrevistas e foco desse estudo foram analisados e interpretados com auxílio do programa Microsoft Excel for Windows XP.

\section{Resultados}

A partir dos dados recolhidos, foi possível perceber que existiam, em 2009, 45 alunos com deficiência matriculados e frequentes em cursos de graduação presencial nas IES pública e privadas. Ao analisar esse resultado por número total absoluto e relativo de pessoas com deficiência em processo de inclusão e por IES, percebeu-se que a pública apresentava oito alunos com deficiência (0,07\%) em um total de 11.000 alunos matriculados em 45 cursos. As privadas possuíam, à mesma época, 37 alunos com deficiência $(0,17 \%)$ em um total de 22.631 alunos matriculados em 85 cursos disponíveis. Foi possível perceber que o número de alunos com deficiência matriculados por IES privadas variou de zero a 12 (1,3\%). Constatou-se assim que, em relação ao total de estudantes que cada categoria administrativa de instituição recebe, o número de alunos em processo de inclusão foi maior nas IES privadas (Tabela 1).

Tabela 1 - Dados referentes à inclusão de pessoas com deficiência por IES pública e privadas.

\begin{tabular}{l|c|c|c|c}
\hline Universidade/ Faculdade & Alunos matriculados & Cursos & $\begin{array}{c}\text { Alunos com defi- } \\
\text { ciência }\end{array}$ & $\begin{array}{c}\% \text { alunos com defi- } \\
\text { ciência }\end{array}$ \\
\hline IES Pública & 11.000 alunos & 35 & 8 & $0,07 \%$ \\
\hline & 4.000 alunos & 14 & 12 & $0,30 \%$ \\
& 2.000 alunos & 6 & 7 & $0,35 \%$ \\
& 3.000 alunos & 12 & 5 & $0,16 \%$ \\
IES Privadas & 1.200 alunos & 6 & 3 & $0,25 \%$ \\
& 1.900 alunos & 6 & 3 & $0,15 \%$ \\
& 230 alunos & 1 & 3 & $1,30 \%$ \\
& 4.400 alunos & 17 & 3 & $0,06 \%$ \\
& 765 alunos & 4 & 1 & $0,13 \%$ \\
TOTAL & 1.136 alunos & 5 & - & $0 \%$ \\
\hline
\end{tabular}

Fonte: Ferreira; Duarte (2010).

LEGENDA: IES - Instituição de Ensino Superior

Acredita-se que este fato se deve a aspectos tais como: o acesso, via processo seletivo, mais disputado e falta de política de cotas de vagas para pessoas com deficiência na IES pública; a maior disponibilidade de cursos noturnos, a possibilidade de bolsa pelo ProUni e até mesmo acesso facilitado à financiamento estudantil pelo FIES nas redes privadas. Estes valores, 
encontrados em uma realidade local, estáo de acordo com a realidade nacional apresentada pelo Censo da Educação Superior de 2010 (BRASIL, 2012), ou seja, maior tendência de matrícula em cursos noturnos e em IES Privadas (distribuição representada no Gráfico 1).

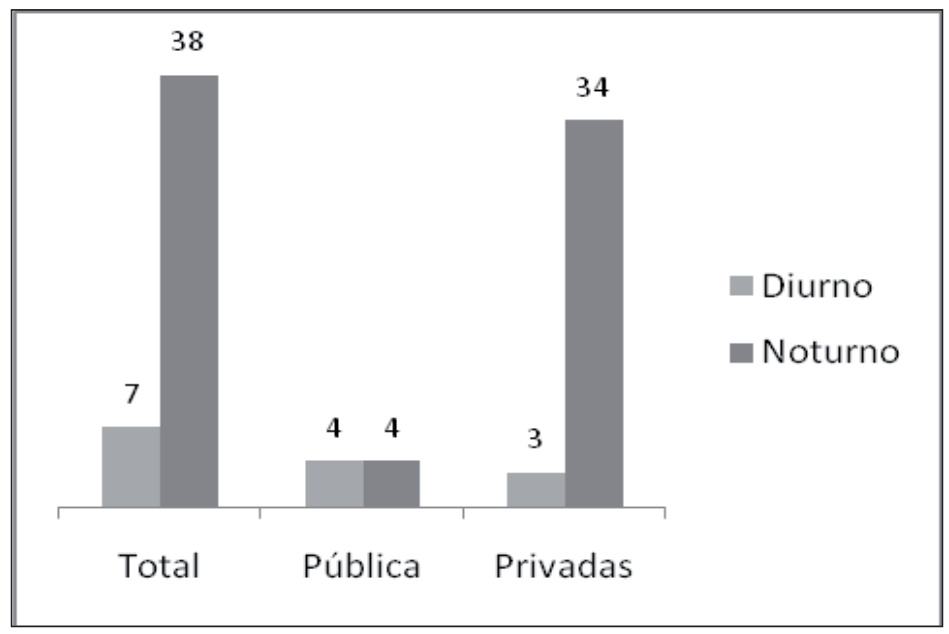

Gráfico 1 - Matriculas de alunos com deficiência por turno de estudo. Fonte: os autores (2013).

Em relação à distribuição dos alunos com deficiência nas áreas de conhecimento, como pode-se perceber no Gráfico 2, a maioria das matrículas concentra-se na área de Humanidades, seguida pela área de Saúde e Exatas. $\mathrm{Na}$ área de Humanidades, os cursos procurados foram: Pedagogia, Direito, Comunicação, Administração, Psicologia, Ciências Contábeis, História e Turismo. Na área da Saúde, Fisioterapia, Educação Física, Medicina e Ciências Biológicas foram os cursos mais frequentados. $\mathrm{Na}$ área de Exatas, havia alunos matriculados nos cursos de Arquitetura, Ciências da Computação, Design Gráfico, Matemática e Sistemas para Internet.

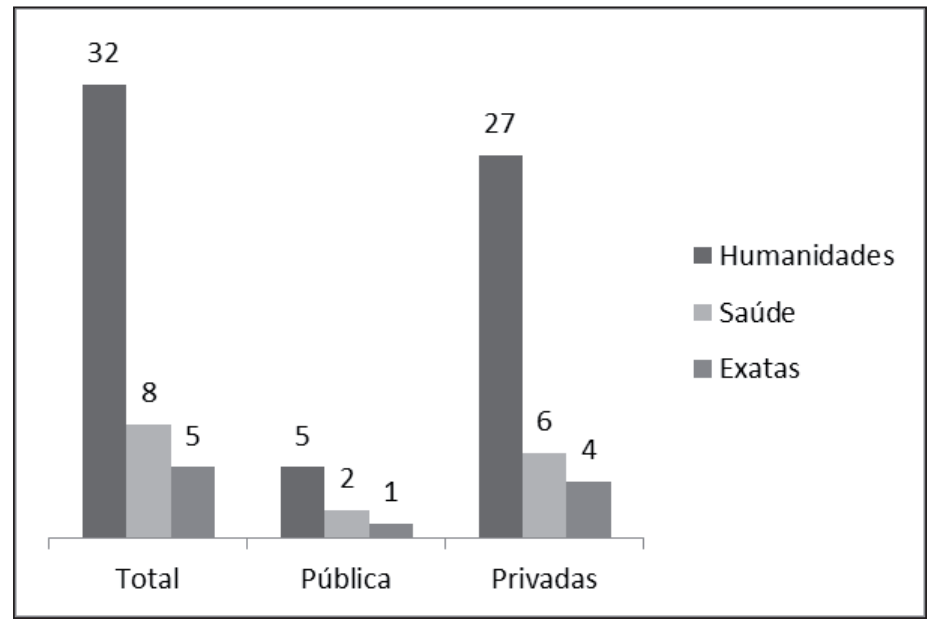

Gráfico 2 - Percentual de matriculas de alunos com deficiência por área de conhecimento. Fonte: os autores (2013). 
Em relação ao sexo, os resultados demonstraram uma distribuição irregular: na rede pública, a maioria dos alunos com deficiência matriculados era do sexo feminino; ao contrário, na rede privada a maioria dos graduandos com deficiência eram do sexo masculino. Ao considerar ambas as redes de ensino, os resultados mantém a tendência observada na rede privada, isto é, maior número de matriculas do sexo masculino.

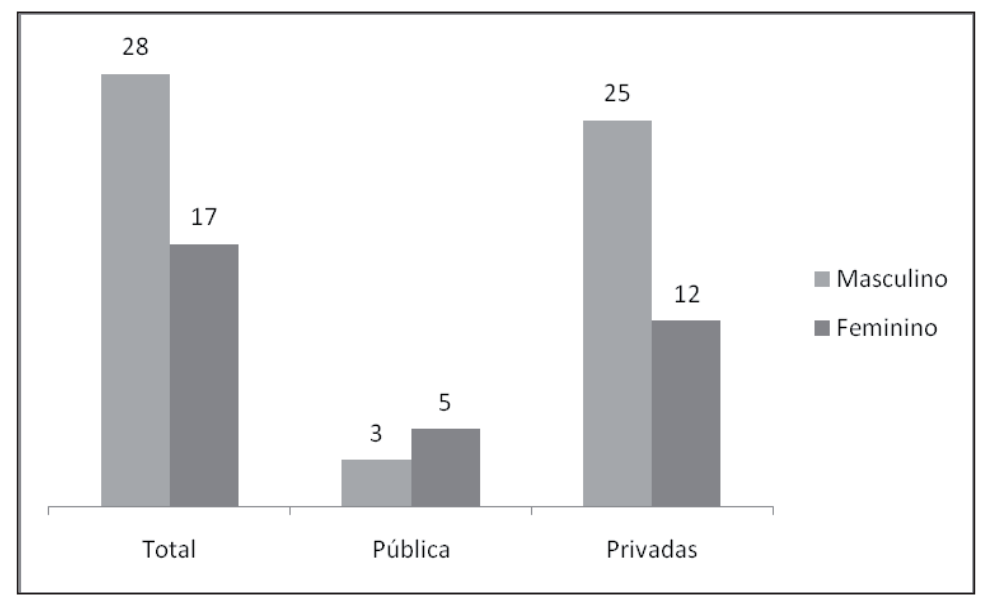

Gráfico 3 - Percentual de matriculas de alunos com deficiência por IES e sexo. Fonte: os autores (2013).

Quando foram analisados os tipos de deficiência, concluiu-se que a deficiência visual apresenta, tanto nas IES privadas quanto na pública, um maior número de matrículas seguido pela deficiência física e auditiva (Gráfico 4).

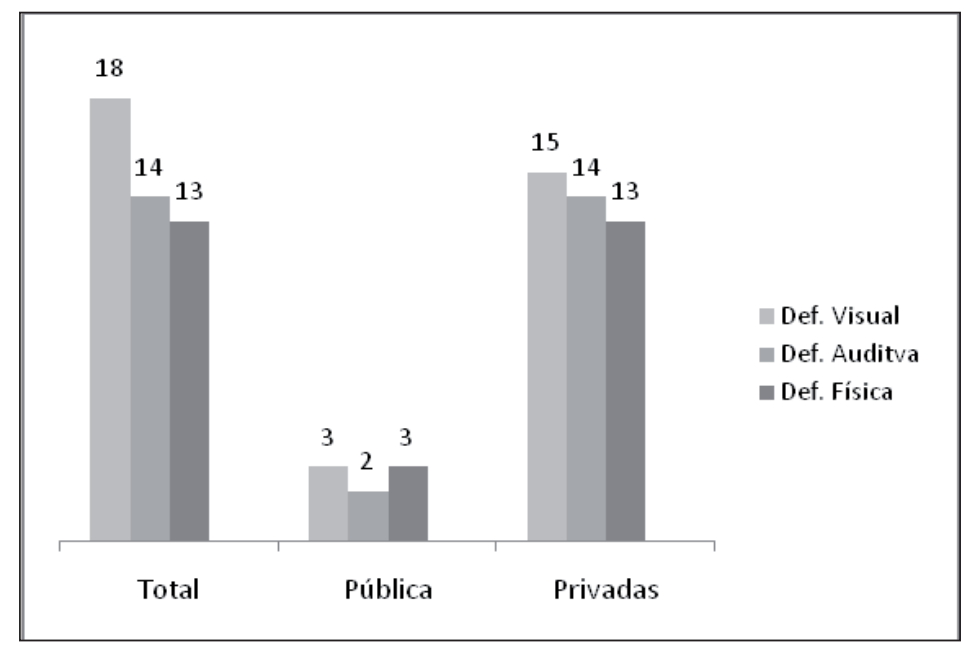

Gráfico 4 - Percentual do total de matriculas de alunos com deficiência por deficiência e por IES. Fonte: os autores (2013). 


\section{CONSIDERAÇÓES FINAIS}

Este estudo teve como propósito relatar a inclusão de pessoas com deficiência no Ensino Superior em Juiz de Fora. A realização de estudos que visem ampliar as informaçóes sobre a inclusão de pessoas com deficiência na rede de ensino brasileira, bem como entender o processo de dificuldades, luta e superaçáo desses estudantes até chegar ao Ensino Superior, tem sido importante para auxiliar os profissionais que estâo envolvidos na luta pelo direito das pessoas com deficiência e o acesso dos mesmos, em especial, no meio educacional.

Sobre o processo de escolarização da pessoa com deficiência no Brasil, cabe aqui não perder de vista a disparidade entre o discurso político de educação para todos e o caráter assistencial e filantrópico que veio ancorando a educação dessas pessoas por um longo período. Mesmo hoje, sob a égide da bandeira inclusiva, são muitos os entraves enfrentados, sobretudo pelos estudantes com deficiência e suas famílias para garantir dignidade e qualidade à sua educação.

Apesar dos últimos dados dos censos escolares indicarem um salto no número de matrículas de alunos com deficiência no ensino regular, o desafio da educação superior para essa população ainda está longe de ser atingida, porque não são apenas os índices quantitativos que precisam ser alterados: todos precisam ter oportunidade de acesso a um ensino de qualidade. Existem leis atuais e modernas a respeito da inclusão. Sabe-se que esses aparatos legais são importantes e necessários à educação para todos nos mais diversos níveis de ensino, mas o problema da sociedade atual está em efetivar a operacionalização dessas leis.

Desse modo, a fim de haver no país uma educação para todos, o gestor do ensino superior público no Brasil precisa investir na produção e distribuição de materiais pedagógicos apropriados, qualificaçáo de professores, infraestrutura adequada para ingresso, acesso e permanência e, nas diversas instituiçóes de ensino, os dirigentes devem estar atentos a qualquer forma de atitude discriminatória. Para tanto, é fundamental a implementação de políticas educacionais que explicitem, em seus programas, de que forma as universidades públicas poderão buscar recursos financeiros para se equiparar adequadamente a essas necessidades.

Como lembram Marques e Marques (2003, p. 237), é função social da universidade "mostrar com clareza as contradiçóes sociais e propor alternativas concretas, pois é nesse contexto que está a perspectiva de incluir". Reafirma-se, a partir deste estudo, a necessidade de realização de outras pesquisas que acompanhem o processo de consolidação da proposta de acesso e permanência no Ensino Superior das pessoas com deficiência.

\section{REFERÊNCIAS}

BRASIL. Instituto Nacional de Estudos e Pesquisas Educacionais Anísio Teixeira Censo da educação superior: 2010 - resumo técnico. Brasília: Instituto Nacional de Estudos e Pesquisas Educacionais Anísio Teixeira, 2012.

CARMO, A. A. C. Aspectos históricos, filosóficos e sociológicos da deficiência. In: FERREIRA, E. L. (Org.). Atividade física para pessoas com deficiência física. Juiz de Fora: ED. UFJF, 2008. 
CHAHINI, T. H. C. O desafio do acesso e permanência dos alunos com necessidades educacionais especiais nas IES de São Luis do Maranhão. 2006. 212f. Dissertação (Mestrado em Educação) - Faculdade de Educação, Universidade Federal do Maranhão, São Luis, 2006.

ONU. Ministério da Educação e Ciência da Espanha. Declaração de Salamanca: e enquadramento da ação na área das necessidades especiais. Salamanca: Espanha, 1994

UNESCO. Declaração mundial de educação para todos. Plano de ação para satisfazer as necessidades básicas de aprendizagem. Tailândia, 1990.

FERREIRA, M. E. C.; DUARTE, E. R. A inclusão de pessoas com deficiência nas instituições de ensino superior e nos cursos de educaçâo física de Juiz de Fora pede passagem. E agora? Juiz de Fora: EDUFJF, 2010.

FERREIRA, M. E. C.; GUIMARÃES, M. Educação Inclusiva. Rio de Janeiro: DP\&A, 2003.

FERREIRA, S. L. Ingresso, permanência e competência: uma realidade possível para universitários com necessidades educacionais especiais. Revista Brasileira de Educação Especial, São Paulo, v.13, n.1, p.43-60, 2007.

GOMES, M. F. C.; LIMA, P. A. L. Inclusão e exclusão: a dupla face da modernidade. In: SEMINÁRIO INTERNACIONAL SOCIEDADE INCLUSIVA, 4., 2006, Belo Horizonte. Anais... Belo Horizonte: 2006. p. 16.

INAJARA, S. M; SANTANA, SILVA, C. Propostas de acessibilidade para a inclusão de pessoas com deficiências no ensino superior. Revista Brasileira de Educação Especial, Marília, v.16, n 1, p 127-136, 2010.

LIMA, O. M. B. A trajetória de inclusão de uma aluna com NEE, Sindrome de Down, no Ensino Superior: um estudo de caso. 2007. 87f. Dissertação (Mestrado Educação) - Faculdade de Educação, Pontifícia Universidade Católica do Rio Grande do Sul, Porto Alegre, 2007.

MACHADO, M, B; TRES, R; OLIVEIRA, L, A. Inserção do deficiente auditivo ou surdo no Ensino Superior da Universidade do Oeste de Santa Catarina - Campus de São Miguel do Oeste. Unoesc \& Ciência-ACHS, Joaçaba, v.2, n.2, p.156-164, 2011.

MANSINI, E.; BAZON, F. A inclusão de estudantes com deficiência no Ensino Superior. São Paulo: Universidade Presbiteriana Mackenzie, 2006.

MANTOAN, M, T, E. (Org.). O desafio das diferenças nas escolas. Petrópolis, RJ: Vozes, 2011.

MARQUES, C. A.; MARQUES, L. P. Do universal ao múltiplo: os caminhos da inclusão. In: OLIVEIRA, M. R. N. S., SOUSA, L. F. E. C. P. (Org.). Politicas educacionais, práticas escolares e alternativas de inclusão escolar. Rio de Janeiro: DP\&A, 2003.

MAZZOTA, M. J. S. Educação especial no Brasil. História e políticas públicas. São Paulo, Cortez, 1996.

MOREIRA, L. C. In(ex)clusão na universidade: o aluno com necessidades educacionais especiais em questão. Revista Educação Especial, Santa Maria, n.25, p.37-47, 2005.

OLIVEIRA, R, B. A escola, a diferença e os sujeitos que nela operam. Monografia de Especialização em Educação Especial: Deficiência Mental e Transtornos e Dificuldades de Aprendizagem junto ao DHEDepartamento de Humanidades e Educação da Universidade Regional do Noroeste do Rio Grande do Sul-UNIJUI. 2012. 
PELLEGRINI, C. M. Ingresso, acesso e permanência dos alunos com necessidades educacionais especiais na Universidade Federal de Santa Maria. 2006. 212f. Dissertação (Mestrado em Educação) - Faculdade de Educação, Universidade Federal de Santa Maria, Santa Maria, 2006.

PEREIRA, M. M. Inclusão e universidade: análise de trajetórias acadêmicas na Universidade Estadual do Rio Grande do Sul. 2007. 201f. Dissertação (Mestrado Educação) - Faculdade de Educação, Universidade Federal do Rio Grande do Sul, Porto Alegre, 2007.

PERINI, T. Í. O processo de inclusão no Ensino Superior em Goiás: a visão dos excluídos. 2006. 121f. Dissertação (Mestrado em Educação) - Faculdade de Educaçáo Faculdade de Educaçáo, Universidade Católica de Goiás, Goiânia, 2006.

REGO, T. C. Memórias de escola: cultura escolar e constituição de singularidades. Petrópolis: Vozes, 2003.

RODRIGUES, D. A inclusão na universidade: limites e possibilidades da construção de uma universidade inclusiva. Cadernos de Educação Especial, Santa Maria, n. 23, 2004. Disponível em: <http://www.ufsm.br/ce/revista/ceesp>. Acesso em: 13 ago. 2009.

SILVA, H. M. et al. A inclusão de estudantes com deficiência no ensino superior: Revisão de literatura. Revista da Universidade Vale do Rio Verde, Três Coraçóes, v.10, n.2, p.332-342, 2012.

VIANNA, L. M. B. P; TARDELLI, P. G. A. S; ALMEIDA, L. I. R. Inclusão e Mercado de Trabalho: uma análise das dificuldades enfrentadas por pessoas com deficiência em ingressar no mercado na Grande Vitória (ES). Revista Destarte, Vitória, v.2, n.2, p.95-109, 2012

Recebido em: 09/08/2012

Reformulado em: 01/05/2013

Aprovado em: 20/05/2013 
DUARTE, E. R. et al. 